Acta Crystallographica Section F

Structural Biology

Communications

ISSN 2053-230X

Toshiharu Tsurumura, * Hao Qiu, Toru Yoshida, Yayoi Tsumori and Hideaki Tsuge

Faculty of Life Sciences, Kyoto Sangyo University, Kamigamo-Motoyama,

Kyoto 603-8555, Japan

Correspondence e-mail:

ttsuru@cc.kyoto-su.ac.jp

Received 8 October 2013

Accepted 30 November 2013

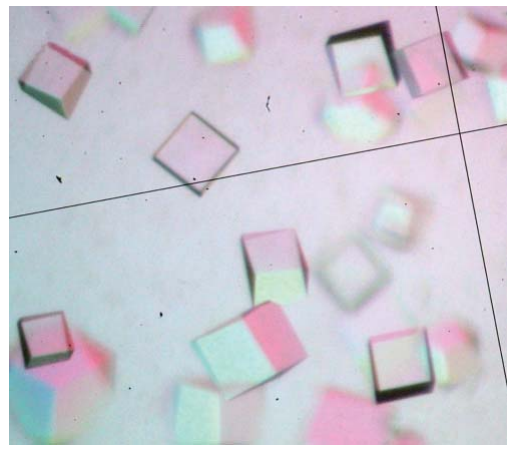

C) 2014 International Union of Crystallography All rights reserved

\section{Crystallization and preliminary X-ray diffraction studies of a surface mutant of the middle domain of PB2 from human influenza $A(H 1 N 1)$ virus}

In the last hundred years, four influenza pandemics have been experienced, beginning with that in Spain in 1918. Influenza A virus causes severe pneumonia and its RNA polymerase is an important target for drug design. The influenza A (H1N1) virus has eight ribonucleoprotein complexes, which are composed of viral RNA, RNA polymerases and nucleoproteins. PB2 forms part of the RNA polymerase complex and plays an important role in binding to the cap structure of host mRNA. The middle domain of PB2 includes a cap-binding site. The structure of PB2 from H1N1 complexed with $\mathrm{m}^{7} \mathrm{GTP}$ has not been reported. Plate-like crystals of the middle domain of PB2 from H1N1 were obtained, but the quality of these crystals was not good. An attempt was made to crystallize the middle domain of PB2 complexed with $\mathrm{m}^{7} \mathrm{GTP}$ using a soaking method; however, electron density for $\mathrm{m}^{7} \mathrm{GTP}$ was not observed on preliminary X-ray diffraction analysis. This protein has hydrophobic residues on its surface and is stable in the presence of high salt concentrations. To improve the solubility, a surface double mutant $(\mathrm{P} 453 \mathrm{H}$ and $\mathrm{I} 471 \mathrm{~T})$ was prepared. These mutations change the surface electrostatic potential drastically. The protein was successfully prepared at a lower salt concentration and good cube-shaped crystals were obtained using this protein. Here, the crystallization and preliminary X-ray diffraction analysis of this mutant of the middle domain of PB2 are reported.

\section{Introduction}

The seasonal influenza virus causes respiratory inflammation, high fever, headache and muscle aches, and fatigue. In the pandemic in 1918, the influenza virus (known as Spanish influenza) also caused severe pneumonia, causing the deaths of 50 million victims worldwide (Reid et al., 2001). Subsequent to this pandemic, humans have been attacked by three further influenza pandemics: Asian influenza (H2N2) in 1957, Hong Kong influenza (H3N2) in 1968 and H1N1 influenza in 2009.

The influenza A virus belongs to the Orthomyxoviridae and has eight viral ribonucleoprotein (vRNP) complexes, which are composed of viral RNA (vRNA), vRNA-dependent RNA polymerases and nucleoproteins (NPs). The vRNP complexes are the basic unit for transcription and replication (Elton et al., 2005). vRNA is a single-stranded negative chain associated with the RNA polymerase and NPs. The RNA polymerase is a heterotrimer composed of three subunits: polymerase acidic protein (PA), polymerase basic protein 1 (PB1) and polymerase basic protein 2 (PB2). This multisubunit complex performs transcription from negative vRNA to positive vRNA and replication of negative vRNA via the replication of complementary DNA.

In the transcription reaction, as a first step PB2 binds the cap structure of host $5^{\prime}$-capped mRNA, such as that of 7-methylguanosine $5^{\prime}$-triphosphate $\left(\mathrm{m}^{7} \mathrm{GTP}\right)$. In the second step, the captured host mRNA is cleaved after 10-13 nucleotides by the endonuclease activity of the PA subunit. Finally, the PB1 subunit plays a central role in the elongation of positive vRNA using the cleaved oligonucleotide as a primer (Plotch et al., 1981).

The PB2 subunit plays a key role in cap binding using its middle domain. Furthermore, PB2 displays a bipartite nuclear localization signal (NLS) in the C-terminal domain and is transported to the 
nucleus (Mukaigawa \& Nayak, 1991). Additionally, residues 627 and 701 in the C-terminal domain of PB2 have been identified to be important factors in host range and pathogenicity (Subbarao et al., 1993; Hatta et al., 2001; Steel et al., 2009). Crystal structures of the C-terminal domain of PB2 have been reported by us and by others (Tarendeau et al., 2008; Kuzuhara et al., 2009). Our structure is the first deposited structure of a PB2 C-terminal domain containing the pathogenicity-determinant lysine residue at position 627 in the Protein Data Bank (PDB entry 3cw4), and we also revealed that the RNA-binding ability of this domain is enhanced by an E627K mutation (Kuzuhara et al., 2009).

The cap-binding site is located in the middle domain of PB2. Crystal structures of this domain from three strains have been reported: human $\mathrm{H} 1 \mathrm{~N} 1 \mathrm{~PB} 2$ middle domain without $\mathrm{m}^{7} \mathrm{GTP}$ (Liu, Meng et al., 2013), human H3N2 PB2 middle domain with $\mathrm{m}^{7} \mathrm{GTP}$ (Guilligay et al., 2008; Liu, Qin et al., 2013) and human H5N1 PB2 middle domain with $\mathrm{m}^{7} \mathrm{GTP}$ (Liu, Qin et al., 2013). However, the structure of the middle domain of PB2 from H1N1 complexed with $\mathrm{m}^{7} \mathrm{GTP}$ has not been reported.

We obtained the human H1N1 PB2 middle-domain protein under high-salt conditions and also obtained crystals. Because the crystals were not of good quality, we collected a data set with considerable difficulty. Although we obtained a crystal of the middle domain of PB2 with $\mathrm{m}^{7}$ GTP by a soaking method using this crystal, electron

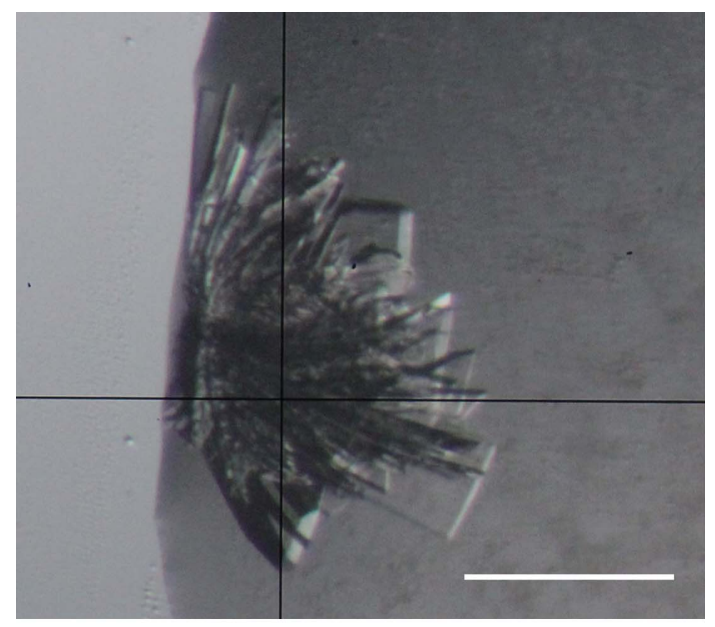

(a)

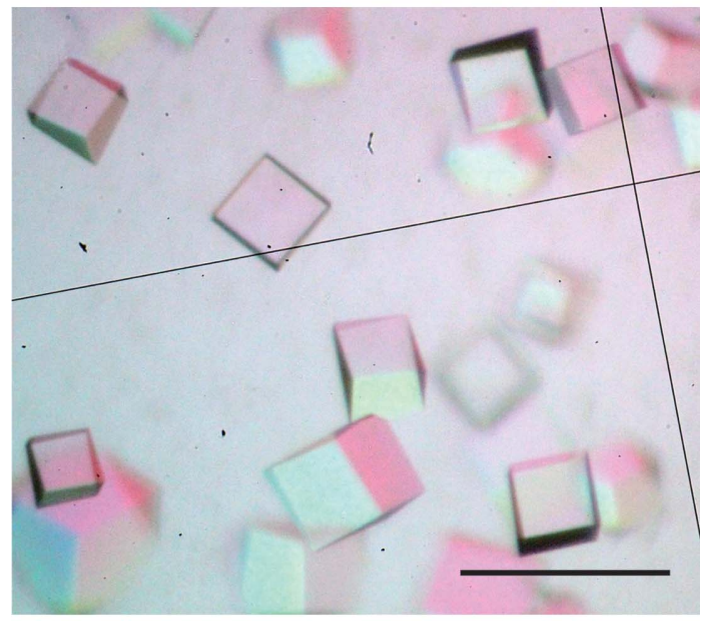

(b)

Figure 1

Crystals of the middle domain of PB2 from H1N1. (a) Wild-type crystals, $(b)$ double-mutant (P453H, I471T) crystals. The scale bars represent $200 \mu \mathrm{m}$. density for $\mathrm{m}^{7} \mathrm{GTP}$ was not observed on preliminary structural analysis. The surface of the PB2 middle-domain molecule tends to be hydrophobic and thus the protein is solubilized in the presence of high salt concentrations. Therefore, we prepared the middle domain of PB2 with mutations of two amino acids (P453H and I471T), resulting in an increase in the solubility of the protein. Here, we report the crystallization and preliminary X-ray diffraction analysis of the PB2 middle-domain mutant.

\section{Materials and methods}

2.1. Cloning, mutation, expression and purification of the middle domain of PB2

The $p b 2$ gene (RIKEN) was inferred from the amino-acid sequence of influenza virus H1N1 (A/Puerto Rico/8/1934). We used DNA encoding residues $318-483$ subcloned into the pET-15b vector between the NdeI and BamHI sites. The PB2 middle domain in pET-15b was mutated using the QuikChange Lightning Multi SiteDirected Mutagenesis Kit (Agilent Technologies) according to the manufacturer's instructions to convert proline to histidine at position 453 using the primer 5'-GGGAGTTGAACATATCGACAATGTG$3^{\prime}$. To convert isoleucine to threonine at position 471 , the $\mathrm{P} 453 \mathrm{H}$ mutant was then mutated using the primers $5^{\prime}$-GACTCCAAGCACCGAGATGTCAATG-3' and 5'-CATTGACATCTCGGTGCTTGGGAGTC-3' using PrimeSTAR HS DNA Polymerase (Takara Bio Inc.) according to the manufacturer's instructions. The middle domain of PB2 was then expressed with an N-terminal histidine tag and a thrombin cleavage site in Escherichia coli BL21-CodonPlusRIL cells (Agilent Technologies) grown in LB medium. The protein was purified by affinity capture on a Protino Ni-IDA 2000 column (Macherey-Nagel). After elution with imidazole, the protein was cleaved with thrombin, loaded onto a Uno S column (Bio-Rad) and eluted with a salt gradient $(0.5-1 \mathrm{M} \mathrm{NaCl})$. The eluted wild-type PB2 middle domain was then concentrated to $10 \mathrm{mg} \mathrm{ml}^{-1}$ in $50 \mathrm{mM}$ Tris$\mathrm{HCl} \mathrm{pH} 8.0,500 \mathrm{~m} M \mathrm{NaCl}, 2 \mathrm{~m} M$ DTT and stored at $-80^{\circ} \mathrm{C}$. The double mutant of the middle domain of $\mathrm{PB} 2$ was purified using the same steps as used for the wild type and was then concentrated to $10 \mathrm{mg} \mathrm{ml}^{-1}$ in $50 \mathrm{~m} M$ Tris- $\mathrm{HCl} \mathrm{pH} 8.0,200 \mathrm{~m} M \mathrm{NaCl}, 2 \mathrm{~m} M$ DTT. The amino-acid sequence of the middle domain of PB2 with two mutations was ${ }^{318}$ RISSSFSFGGFTFKRTSGSSVKREEEVLTGNLQTLKIRVHEGYEEFTMVGRRATAILRKATRRLIQLIVSGRDEQSIAEAIIVAMVFSQEDCMIKAVRGDLNFVNRANQRLNPMHQLLRHFQKDAKVLFQNWGVEHIDNVMGMIGILPDMTPSTEMSMRGVRISKM $^{483}$. The residues in bold indicate the locations of the two mutations. An additional four residues derived from the expression vector were present at both the $\mathrm{N}$-terminus and the C-terminus: ${ }^{314} \mathrm{GSHM}^{317}$ and ${ }^{481} \mathrm{GSGC}^{484}$, respectively.

\subsection{Crystallization, data collection and model building}

Crystals of the wild type were grown using the hanging-drop vapour-diffusion method. The protein solution was mixed with an equal volume of well solution $\left[0.1 M \mathrm{NaH}_{2} \mathrm{PO}_{4}, 0.1 M \mathrm{KH}_{2} \mathrm{PO}_{4}, 0.1 M\right.$ MES pH 5.9, $0.8 \mathrm{M} \mathrm{NaCl}, 4 \%(v / v)$ 1-propanol] and incubated at $4{ }^{\circ} \mathrm{C}$. Crystals were picked up using a nylon loop. They were then dipped into mother liquor containing $20 \%(\mathrm{v} / \mathrm{v})$ glycerol as a cryoprotectant and plunged into a nitrogen-gas stream at $100 \mathrm{~K}$. The data were collected using an X-ray wavelength of $1.0 \AA$ and a Quantum 210r detector on beamline AR-NW12A at the KEK Photon Factory (PF), Tsukuba, Japan. The X-ray data were processed using $H K L-2000$ and the diffraction images were integrated and scaled using $D E N Z O$ and SCALEPACK (Otwinowski \& Minor, 1997). The crystal space group 
Table 1

Data-collection and structure-refinement statistics.

Values in parentheses are for the last resolution shell.

\begin{tabular}{|c|c|c|}
\hline & Wild type & $\begin{array}{l}\mathrm{P} 453 \mathrm{H} / \mathrm{I} 471 \mathrm{~T} \\
\text { double mutant }\end{array}$ \\
\hline \multicolumn{3}{|l|}{ Data collection } \\
\hline Unit-cell parameters $(\AA ̊)$ & $\begin{array}{l}a=75.9, b=103.5 \\
\quad c=112.5\end{array}$ & $\begin{array}{l}a=108.0, b=108.0 \\
\quad c=137.6\end{array}$ \\
\hline Space group & $I 2_{1} 2_{1} 2_{1}$ & $R 32$ \\
\hline Beamline & AR-NW12A, PF & MicroMax-007 HF \\
\hline Detector & Quantum 210r & R-AXIS VII \\
\hline Data-collection range $\left({ }^{\circ}\right)$ & $0-180$ & $0-240$ \\
\hline Oscillation $\left(^{\circ}\right)$ & 1 & 0.5 \\
\hline Exposure time (s) & 1 & 60 \\
\hline Mosaicity $\left({ }^{\circ}\right)$ & 1.486 & 0.585 \\
\hline Resolution (§) & $50.0-2.40(2.48-2.40)$ & $50.0-2.30(2.38-2.30)$ \\
\hline Total No. of reflections & $116427(10541)$ & 99657 (9611) \\
\hline No. of unique reflections & $16436(1495)$ & $13943(1367)$ \\
\hline Completeness (\%) & $92.4(85.4)$ & $100.0(100.0)$ \\
\hline$R_{\text {merge }} \dagger$ & $0.061(0.465)$ & $0.093(0.680)$ \\
\hline$R_{\text {meas }} \hbar$ & $0.066(0.502)$ & $0.100(0.734)$ \\
\hline$R_{\text {p.i.m. }} \S$ & $0.025(0.187)$ & $0.037(0.276)$ \\
\hline$\langle I / \sigma(I)\rangle$ & $27.1(4.7)$ & $21.4(4.1)$ \\
\hline Multiplicity & $7.1(7.1)$ & $7.1(7.0)$ \\
\hline \multicolumn{3}{|l|}{ Refinement } \\
\hline$R_{\text {work }} \Phi$ & 0.218 & \\
\hline$R_{\mathrm{free}} \dagger \dagger$ & 0.271 & \\
\hline R.m.s.d., bond lengths $(\AA)$ & 0.015 & \\
\hline R.m.s.d., bond angles $\left({ }^{\circ}\right)$ & 1.787 & \\
\hline Matthews coefficient $\left(\AA^{3} \mathrm{Da}^{-1}\right)$ & 2.99 & \\
\hline No. of molecules in asymmetric unit & 2 & \\
\hline Solvent content $(\%)$ & 58.8 & \\
\hline No. of atoms in the model & 2582 & \\
\hline No. of water molecules & 71 & \\
\hline $\begin{array}{l}\text { No. of residues in outlier region } \\
\text { of Ramachandran plot }\end{array}$ & 0 & \\
\hline Wilson $B$ factor $\left(\AA^{2}\right)$ & 51.1 & \\
\hline Average $B$ factor $\left(\AA^{2}\right)$ & 58.0 & \\
\hline
\end{tabular}

$\dagger R_{\text {merge }}=\quad \sum_{h k l} \sum_{i}\left|I_{i}(h k l)-\langle I(h k l)\rangle\right| / \sum_{h k l} \sum_{i} I_{i}(h k l) . \quad \neq R_{\text {mea }}$ $\left.\sum_{h k l}\{N(h k l) /[N(h k l)-1]\}\right\}^{1 / 2} \sum_{i}\left|I_{i}(h k l)-\langle I(h k l)\rangle\right| / \sum_{h k l} \sum_{i} I_{i}(h k l) . \quad \S R_{\text {p.i.m. }}$ $\sum_{h k l}\{1 /[N(h k l)-1]\}^{1 / 2} \sum_{i}\left|I_{i}(h k l)-\langle I(h k l)\rangle\right| / \sum_{h k l} \sum_{i} I_{i}(h k l)$, where $I_{i}(h k l)$ is the $i$ th intensity measurement of reflection $h k l,\langle I(h k l)\rangle$ is the mean intensity for this reflection and $N(h k l)$ is the multiplicity. $-R_{\text {work }}=\sum_{h k l}|| F_{\text {obs }}|-| F_{\text {calc }}|| / \sum_{h k l}\left|F_{\text {obs }}\right|$. $\dagger \dagger R_{\text {free }}$ was calculated using randomly selected reflections $(5 \%)$.

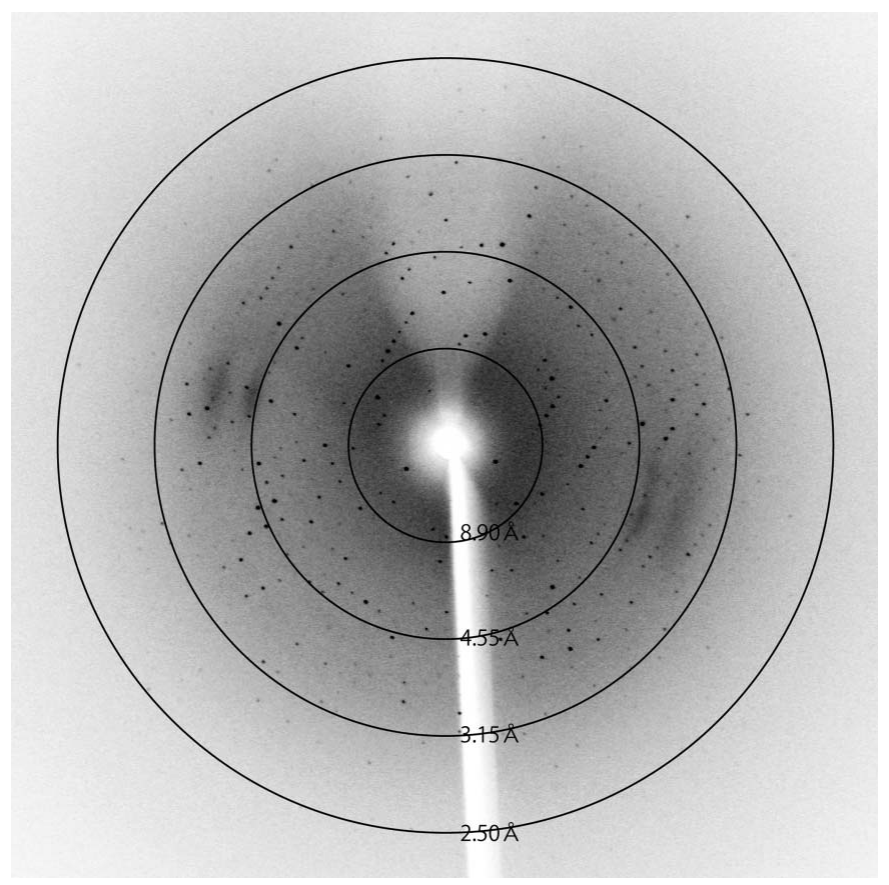

Figure 2

A representative $0.5^{\circ}$ oscillation image of data collected from a crystal of the PB2 middle-domain double mutant using an R-AXIS VII detector in our laboratory. was determined to be $I 2_{1} 2_{1} 2_{1}$. The initial phase was obtained by molecular replacement (MOLREP in CCP4; Vagin \& Teplyakov, 2010; Winn et al., 2011) using the H3N2 cap-binding domain structure (PDB entry 2vqz; Guilligay et al., 2008) as a model. The structure was refined using REFMAC5 restrained refinement in CCP4 (Murshudov et al., 2011) and the rebuilding process was performed using Coot (Emsley \& Cowtan, 2004).

For the double mutant of the middle domain of $\mathrm{PB} 2$, the protein solution was mixed with an equal volume of well solution $[1.2 \mathrm{M}$ $\mathrm{NaCl}, 2.5 \%(v / v)$ ethanol] and crystals were grown using the hangingdrop vapour-diffusion method at $4^{\circ} \mathrm{C}$. Data collection was carried out in the same way as for the wild type except that mother liquor containing $20 \%(v / v)$ ethylene glycol was used as a cryoprotectant. The preliminary data were collected using an X-ray wavelength of $1.54 \AA$ and an R-AXIS VII detector system on a MicroMax-007 HF generator (Rigaku) in our laboratory. The X-ray data were processed using the same steps as used for the wild type. The crystal space group was determined to be $R 32$.

\section{Results and discussion}

We purified His-tagged middle domain of PB2 from H1N1 using a nickel chelate column and the protocol reported by Guilligay et al. (2008). However, aggregation of the protein was observed in $200 \mathrm{mM}$ $\mathrm{NaCl}$ solution. The middle domain of PB2 from H1N1 thus appears to be less soluble than the middle domain of PB2 from H3N2. We modified the protocol to elute the protein with $500 \mathrm{mM} \mathrm{NaCl}$ and found that the middle domain of PB2 from H1N1 was stable under these high-salt conditions. Upon crystallization, clustered plate-like crystals appeared within $2-5 \mathrm{~d}$ under high-salt conditions (Fig. 1a). We collected a preliminary data set to $2.42 \AA$ resolution from a single crystal and found that the space group was $I 22_{1} 2_{1} 2_{1}$, with unit-cell parameters $a=75.9, b=103.5, c=112.5 \AA$. Molecular-replacement analysis was performed with $M O L R E P$ in $C C P 4$ and showed that two PB2 middle-domain molecules were located in the asymmetric unit. The Matthews coefficient value (Matthews, 1968) was $2.99 \AA^{3} \mathrm{Da}^{-1}$ and corresponds to a solvent content of approximately $60 \%$. The $R_{\text {work }}$ and $R_{\text {free }}$ values for the initial solution of two PB2 middledomain molecules were 45.0 and $43.2 \%$, respectively. Refinement and manual fitting of the model resulted in a final $R_{\text {work }}$ of $21.8 \%$ and $R_{\text {free }}$ of $27.1 \%$. Other statistics of data collection and structure refinement are given in Table 1. The structure was deposited in the PDB as entry $4 \mathrm{j} 2 \mathrm{r}$. We attempted a soak with $\mathrm{m}^{7} \mathrm{GTP}(2 \mathrm{mM}, 30 \mathrm{~min})$ using this crystal, but we were unable to observe any electron density for $\mathrm{m}^{7}$ GTP.

Therefore, we produced the middle domain of PB2 with two mutations ( $\mathrm{P} 453 \mathrm{H}$ and $\mathrm{I} 471 \mathrm{~T})$ on the surface. These mutations, which are not near the cap-binding site, change the electrostatic surface from hydrophobic to hydrophilic and resulted in an improvement in the solubility. We screened the crystallization conditions of the mutant in the apo state and obtained crystals under different conditions to those used for the wild type. The crystal shape was improved: a single thick cube-shaped crystal (Fig. 1b) appeared within one week. We tested the diffraction using a small crystal $(<0.1 \mathrm{~mm})$ and collected a preliminary X-ray diffraction data set at $2.30 \AA$ ̊ resolution using a MicroMax-007 HF rotating-anode X-ray generator (Rigaku; Fig. 2). The crystal space group was determined to be $R 32$, with unit-cell parameters $a=107.8, b=107.8, c=136.6 \AA$. The statistics of data collection are described in Table 1 . The crystals usually grew to $0.4 \mathrm{~mm}$ in size and we can therefore expect to obtain good data sets using a synchrotron light source. 
In summary, we improved the solubility of the middle domain of PB2 using two mutations and succeeded in the crystallization of this mutant. Using the double surface mutant crystals, we are now attempting to obtain high-resolution data using a synchrotron light source in order to reveal the substrate-binding mechanism of $\mathrm{m}^{7} \mathrm{GTP}$ in the middle domain of PB2 from the H1N1 strain.

This work was supported in part by a Strategic Research Foundation Grant-aided Project for Private Universities and a Grant-inAid for Scientific Research on Innovative Areas, MEXT/JSPS KAKENHI Grant No. 25121733, Japan. The influenza virus (A/PR/8/ 34) RNA polymerase PB2 plasmid, pBMSA-PB2, was provided by the DNA Bank, RIKEN BioResource Center, Tsukuba, Japan (originally deposited by Dr Susumu Nakada) with the support of the National Bio-Resources Project of MEXT. We thank the staff at KEK $\mathrm{PF}$ for data collection.

\section{References}

Elton, D., Digard, P., Tiley, L. \& Ortin, J. (2005). Influenza Virology: Current Topics, edited by Y. Kawaoka, pp. 1-36. Wymondham: Caister Academic Press.

Emsley, P. \& Cowtan, K. (2004). Acta Cryst. D60, 2126-2132.
Guilligay, D., Tarendeau, F., Resa-Infante, P., Coloma, R., Crepin, T., Sehr, P., Lewis, J., Ruigrok, R. W., Ortin, J., Hart, D. J. \& Cusack, S. (2008). Nature Struct. Mol. Biol. 15, 500-506.

Hatta, M., Gao, P., Halfmann, P. \& Kawaoka, Y. (2001). Science, 293, 18401842.

Kuzuhara, T., Kise, D., Yoshida, H., Horita, T., Murazaki, Y., Nishimura, A., Echigo, N., Utsunomiya, H. \& Tsuge, H. (2009). J. Biol. Chem. 284, 68556860.

Liu, Y., Meng, G., Luo, M. \& Zheng, X. (2013). Acta Cryst. F69, 280-283.

Liu, Y., Qin, K., Meng, G., Zhang, J., Zhou, J., Zhao, G., Luo, M. \& Zheng, X. (2013). J. Biol. Chem. 288, 11013-11023.

Matthews, B. W. (1968). J. Mol. Biol. 33, 491-497.

Mukaigawa, J. \& Nayak, D. P. (1991). J. Virol. 65, 245-253.

Murshudov, G. N., Skubák, P., Lebedev, A. A., Pannu, N. S., Steiner, R. A., Nicholls, R. A., Winn, M. D., Long, F. \& Vagin, A. A. (2011). Acta Cryst. D67, 355-367.

Otwinowski, Z. \& Minor, W. (1997). Methods Enzymol. 276, 307-326.

Plotch, S. J., Bouloy, M., Ulmanen, I. \& Krug, R. M. (1981). Cell, 23, 847-858.

Reid, A. H., Taubenberger, J. K. \& Fanning, T. G. (2001). Microbes Infect. 3, 81-87.

Steel, J., Lowen, A. C., Mubareka, S. \& Palese, P. (2009). PLoS Pathog. 5, e1000252.

Subbarao, E. K., Kawaoka, Y. \& Murphy, B. R. (1993). J. Virol. 67, 7223-7228.

Tarendeau, F., Crepin, T., Guilligay, D., Ruigrok, R. W., Cusack, S. \& Hart, D. J. (2008). PLoS Pathog. 4, e1000136.

Vagin, A. \& Teplyakov, A. (2010). Acta Cryst. D66, 22-25.

Winn, M. D. et al. (2011). Acta Cryst. D67, 235-242. 